\title{
Osteoporosis
}

\section{Pyrrolidine-Containing Bisphosphonates as Potential Anti-Resorption Bone Drugs}

\author{
Lorena De Luca, Andrea Chiminazzo, Laura Sperni, Giorgio Strukul, and Alessandro Scarso*[a]
}

Abstract: Bisphosphonates, particularly those with N-substituted groups, are currently the most popular drugs for the treatment of osteoporosis. However, their chemical structures are still rather simple and new synthetic methods are needed to expand their molecular complexity and also improve their specificity of action towards other targets as anticancer, antibacterial, and antimalarial drugs. Herein, we report a new class of potential antiresorption bisphospho- nate drugs that have a pyrrolidine unit with different substituents, obtained through a simple dipolar cycloaddition reaction between azomethine ylides and vinylidenebisphosphonate derivatives as precursors. The methodology led to the efficient preparation of a wide range of (1-methylpyrrolidine-3,3-diyl)bis(phosphonic esters) derivatives with different substituents in position 4.

\section{Introduction}

Because of their similarity to pyrophosphate, which is an important constituent of hydroxyapatite (HAP), the major constituent of the mineral portion of bones, bisphosphonates (BPs) are widely employed as drugs for the treatment of bone disorders, such as hypocalcaemia and osteoporosis (Figure 1). By exploiting their specific bone-targeting properties, ${ }^{[1]}$ this class of molecules has been used for decades in medical treatments, ${ }^{[2]}$ although the correlation between their chemical structure and biological activity is a still debated topic. ${ }^{[3,4]}$ The mineral portion of bones is constantly formed by osteoblasts and redissolved by osteoclasts. The cellular activity of BPs is related to their ability to inhibit specific enzymes, such as farnesyl diphosphate synthase (FPPS), geranylgeranyl diphosphate synthase (GGPPS), ${ }^{[5,6]}$ and others, ${ }^{[7]}$ present in osteoclasts, which leads to their inactivation and eventual apoptosis.

In the chemical structure of BPs, the presence of a hydroxyl group in position $\mathrm{R}^{1}$ (Figure 1) enhances the affinity for HAP and the presence of $\mathrm{N}$-substituted residues on $\mathrm{R}^{2}$ promotes good performance in terms of antiresorptive efficiency, as demonstrated, for example, by alendronic and zoledronic acid, which are sold as drugs to combat osteoporosis (Figure 1). High activity has been recently observed with a new class of more-lipophilic BPs that lack the presence of the gem-OH group in position $\mathrm{R}^{1}$ but have $\mathrm{N}$-substituted cationic residues with a long alkyl chain on $\mathrm{R}^{2}$. These molecules proved to be extremely potent for osteoclast inhibition because they targeted both enzymes, ${ }^{[5]}$ and they also have good prospects as anti-

\footnotetext{
[a] L. De Luca, Dr. A. Chiminazzo, Dr. L. Sperni, Prof. G. Strukul, Prof. A. Scarso Dipartimento di Scienze Molecolari e Nanosistemi

Università Ca' Foscari Venezia, via Torino 155, 30170 Venezia Mestre (Italy) E-mail:alesca@unive.it

$\square$ Supporting information for this article and $O R C I D(s)$ for the author(s) can be found under: http://dx.doi.org/10.1002/chem.201605878.
}

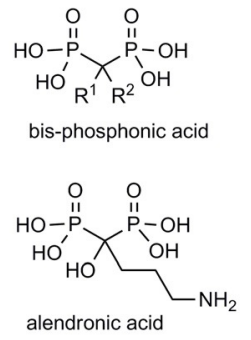

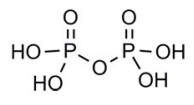

pyrophosphoric acid

$$
\text { alendronic acid }
$$

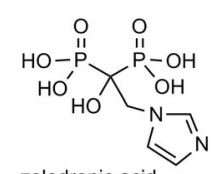

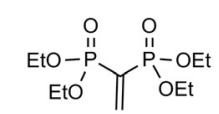

vinylidenebisphosphonate ethyl ester

Figure 1. Chemical structures of bisphosphonates, pyrophosphoric acid, alendronic and zoledronic acids, and vinylidenebisphosphonate tetraethyl ester.

cancer, ${ }^{[8]}$ antibacterial, and antimalarial drugs. ${ }^{[9]}$ The development of new bisphosphonate drugs with $\mathrm{N}$ residues on $\mathrm{R}^{2}$ is a highly desirable topic in the search for active drug candidates with fewer side effects typical of this class of molecules, such as inflammatory symptoms like fever, myalgia, arthralgias and headache.

The synthesis of BPs differs if the target molecule has $\mathrm{R}^{1}=$ $\mathrm{OH}$ or $\mathrm{H}$. In the first case, BPs are usually prepared by starting from an acyl chloride ${ }^{[10]}$ or a carboxylic acid. ${ }^{[11,12]}$ Conversely, BPs with $\mathrm{R}^{1}=\mathrm{H}$ are generally prepared by conjugate addition of nucleophiles to vinylidenebisphosphonate tetraethylester $(\mathrm{VBP})^{[13]}$ as a typical building block. Through this method, BPs with heterocycles, ${ }^{[14]}$ steroid conjugates, ${ }^{[15]}$ or other functional groups at the $\beta$ position, such as thiols, ${ }^{[16]}$ have been reported in the literature. General classes of BPs have also been prepared by metal-catalyzed addition of boronic acids and indoles to VBP, as recently disclosed by our group. ${ }^{[17,18]}$ Nitrogen-substituted BPs can be obtained with a multitude of functional 
groups. For example, VBP acts as an efficient dienophile ${ }^{[19]}$ for cycloaddition reactions with dienes ${ }^{[20]}$ and nitrones ${ }^{[21]}$ to provide cyclic BPs and isoxazolidine-substituted BPs, respectively. BPs with two alkyl derivatives in positions $R^{1}$ and $R^{2}$, or one alkyl and one alkoxy derivative, could be obtained through the formation of a new $\mathrm{C}-\mathrm{P}$ bond by starting from monophosphonates. $^{[22]}$

\section{Results and Discussion}

Herein, we take inspiration from examples of enantioselective 1,3-dipolar cycloadditions of azomethine ylides to electronpoor alkenes, ${ }^{[23]}$ and describe the synthesis of a large series of substituted pyrrolidine that contain bisphosphonates obtained through a 1,3-dipolar cycloaddition of azomethine ylides ${ }^{[24]}$ to VBP and other prochiral BP analogues as dipolarophiles. BPs with similar structures have only marginally been reported in the literature. ${ }^{[19,25]}$

Initially, the reaction between sarcosine, paraformaldehyde, and VBP was investigated to observe the quantitative formation of the corresponding tetraethyl(1-methylpyrrolidine-3,3diyl)bisphosphonate derivative by heating the mixture at reflux in toluene for $5 \mathrm{~h}$ (Scheme 1). The isolated product was charac-

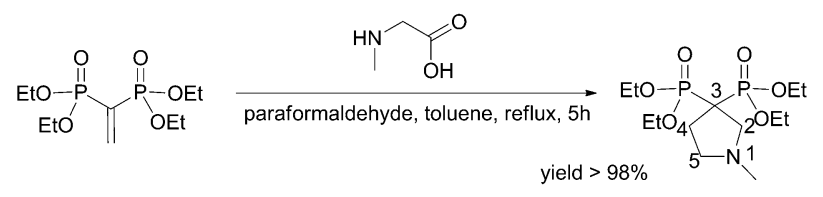<smiles>[R]NC([R])C(=O)OCC</smiles><smiles></smiles>

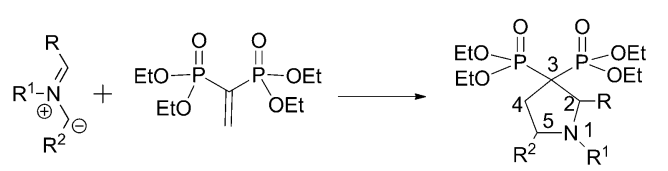

Scheme 1. Synthesis of the 1-methylpyrrolidine-substituted BP by a reaction between sarcosine, paraformaldehyde, and VBP and the mechanism of the formation of the azomethine ylide and its cycloaddition to VBP.

terized by ${ }^{1} \mathrm{H},{ }^{31} \mathrm{P}$, and ${ }^{13} \mathrm{C}$ NMR spectroscopy and GC-MS (see the Supporting Information) and showed a single resonance in the ${ }^{31} \mathrm{P}$ NMR spectrum at $\delta=25.34 \mathrm{ppm}$ due to the presence of a plane of symmetry in the molecule.

Attempts were made to replace sarcosine with different amino acids in the above reaction. Under experimental conditions identical to those in Scheme 1, glycine led to the formation of the corresponding tetraethyl(pyrrolidine-3,3-diyl)bisphosphonate derivative in quantitative yield, which was characterized by a singlet at $\delta=25.24 \mathrm{ppm}$ in the ${ }^{31} \mathrm{P}$ NMR spec- trum (for full characterization, see the Supporting Information). The use of other natural amino acids showed that the reaction is highly influenced by the substituents: alanine and valine resulted in significantly lower product formation (27 and 26\% ${ }^{31} \mathrm{P}$ NMR spectroscopic yield, respectively), whereas amino acids with more branched and longer substituents (e.g., phenylalanine, proline, glutamic acid, and isovaline) showed no evidence of pyrrolidine BP product formation, due to either the limited solubility of the amino acid in the reaction medium or the formation of numerous byproducts. It is worth noting that when chiral amino acids were used, the corresponding pyrrolidine BP products were characterized by the presence of a stereocenter on the C2 carbon atom, as confirmed by the observation of two diastereotopic ${ }^{31} \mathrm{P}$ resonances due to the asymmetry of the molecules (e.g., see the product from alanine with ${ }^{31} \mathrm{P}$ resonances at $\delta=26.65$ and $25.32 \mathrm{ppm}, J=$ $14.6 \mathrm{~Hz}$ ). However, despite starting from natural enantiopure amino acids, the BP products were obtained as a racemic mixture. This is due to the loss of the original stereocenter in the decarboxylation step of the reaction, which leads to the formation of a 1,3-dipole that subsequently adds to the VBP. ${ }^{[26]}$

Substituted prochiral VBP precursors with aromatic units were used in the same reaction in combination with different amino acids and aldehydes to obtain multi-substituted pyrrolidine BP products (Table 1).

Initially, the phenyl and $p$-nitrophenyl precursors were used as dipolarophiles in a 1,3-cycloaddition with glycine and form-

Table 1. 1,3-Cycloaddition between substituted aromatic VBP precursors, different aldehydes, and amino acids. ${ }^{[a]}$

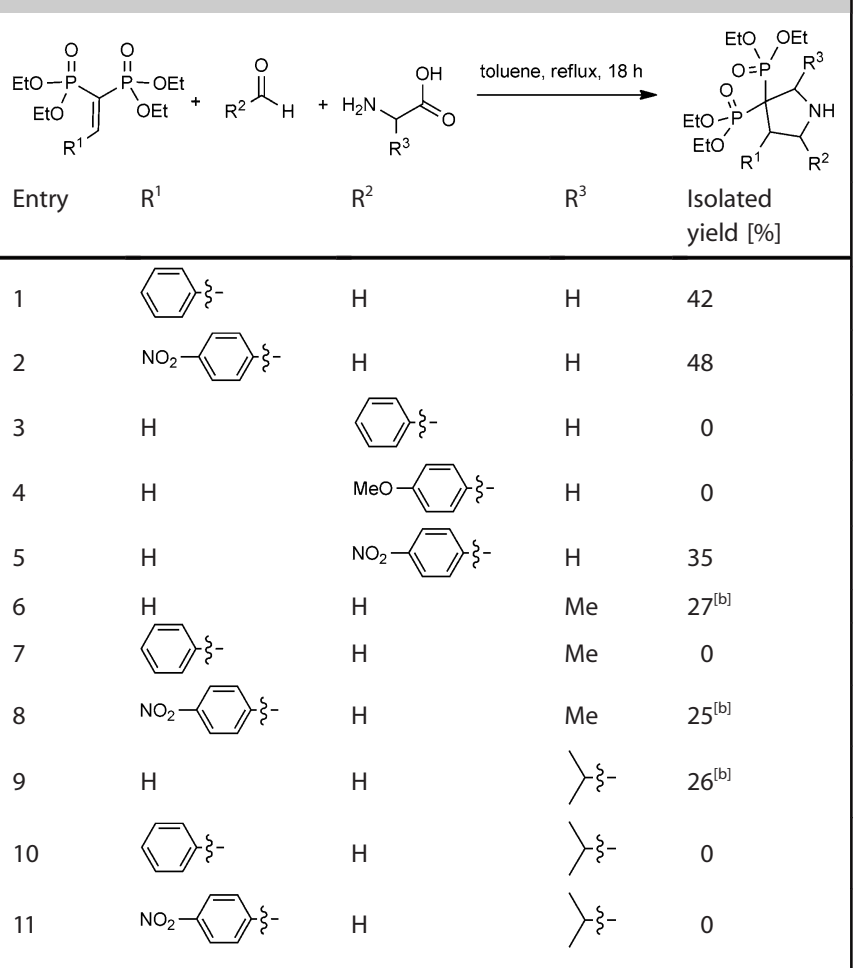

[a] Reaction conditions: BP $(0.65 \mathrm{mmol})$, amino acid $(2.5 \mathrm{mmol})$, aldehyde (6.5 mmol), toluene $(25 \mathrm{~mL}), T=110^{\circ} \mathrm{C}, t=18 \mathrm{~h}$. [b] ${ }^{31} \mathrm{P}$ NMR spectroscopy yield. 
aldehyde, and showed a decrease in reaction yield compared with simple VBP (Table 1, entries 1-2). Compared with simple VBP, these substituted VBP precursors did not lead to the corresponding cycloaddition products in combination with valine (Table 1, entries 9-11), and the reaction with alanine led to similar product formation only with the $p$-nitrophenyl precursor (Table 1, entries 6-8). Although the use of VBP precursors with an electron-withdrawing group (EWG) generally improved reaction yields, probably because they are better dipolarophiles, the results reported in Table 1 generally indicate that steric hindrance plays a fundamental role in this reaction. In fact, several attempts at cycloaddition made with the least bulky amino acid, glycine, with different aromatic aldehydes occurred successfully only with the highly electron-poor $p$-nitrobenzaldehyde in moderate yields, whereas the use of simple benzaldehyde or $p$-OMe-substituted benzaldehyde (Table 1, entries 3 and 4) resulted in a mixture of inseparable byproducts. In general, the obtained products were difficult to purify by using chromatography, probably because of the presence of residual polar non-BP reagents used in the reaction.

Better results for product formation and purification were obtained by using the $\mathrm{N}$-substituted amino acid sarcosine as the reagent, as reported in Table 2 . In all cases, the corresponding 1-methylpyrrolidine 4-substituted BPs were obtained in

Table 2. Synthesis of four-substituted 1-methylpyrrolidine-substituted BPs by treatment of sarcosine with paraformaldehyde and prochiral VBPs. ${ }^{[a]}$

$$
\text { Entry }
$$

yield [\%]

(

[a] Reaction conditions: BP $(0.65 \mathrm{mmol})$, amino acid $(2.5 \mathrm{mmol})$, aldehyde (6.5 mmol), toluene $(25 \mathrm{~mL}), T=110^{\circ} \mathrm{C}, t=18 \mathrm{~h}$. good-to-moderate yields depending on the electronic and steric properties of the prochiral VBP substrate. In fact, as reported in Table 2, the reaction led to a $45 \%$ yield in the case of the phenyl derivative (Table 2 , entry 1 ), whereas the yields were lower with more sterically demanding 2-naphthyl, biphenyl, and o-bromophenyl derivatives (Table 2, entries 2, 3, $10)$, and no products were detected for the $p$-tBu aliphatic VBP derivative (Table 2, entry 5). Similarly, with electron-donating substituents on the aromatic moiety the reaction was less efficient, as in the cases of $t \mathrm{Bu}$-, methoxy- or 3,4-dimethoxyphenyl substituents (Table 2 , entries $4,6,7$ ). Only the $p$-nitrophenyl precursor led to good product formation ( $68 \%$ yield), whereas the $p$-fluorophenyl derivative showed only limited activity and gave an $11 \%$ yield (Table 2 , entries 9,11 ).

The isolated products were characterized by using ${ }^{1} \mathrm{H},{ }^{31} \mathrm{P}$, and ${ }^{13} \mathrm{C}$ NMR spectroscopy and GC-MS. In Figure 2, a typical characterization for the binaphthyl BP derivative is reported. The ${ }^{31} \mathrm{P}\left\{{ }^{1} \mathrm{H}\right\}$ NMR spectrum shows two distinct resonances for

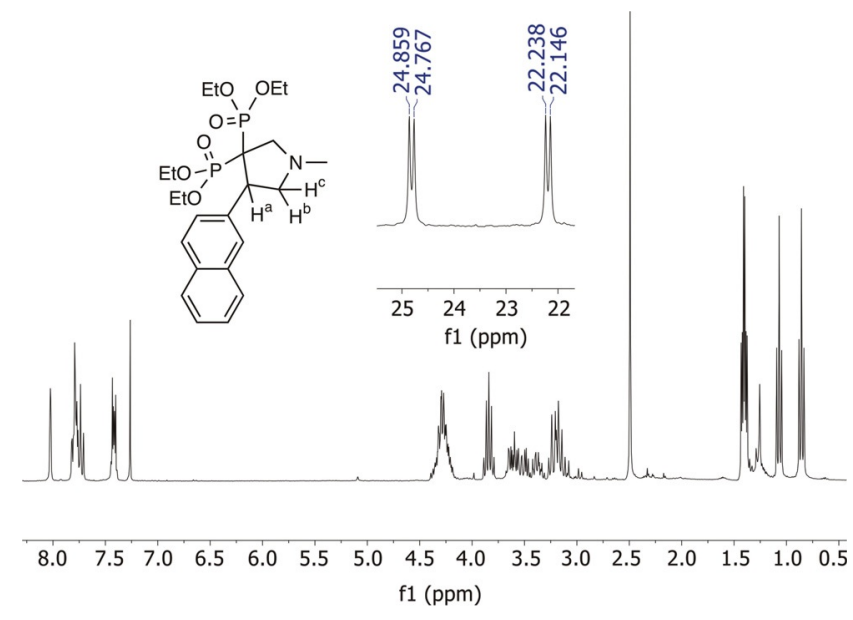

Figure 2. ${ }^{1} \mathrm{H}$ NMR (bottom) and ${ }^{31} \mathrm{P}\left\{{ }^{1} \mathrm{H}\right\}$ NMR spectra (top) of the binaphthyl $\mathrm{BP}$ derivative.

the diastereotopic $\mathrm{P}$ atoms because of the presence of a stereogenic center in the molecule in position 4 . The asymmetry of the molecule was also revealed by the ${ }^{1} \mathrm{H}$ NMR spectrum, in which four different resonances for the terminal methyl units of the ethoxy phosphonate groups were observed. Similar resonances were also observed for the other pyrrolidine BP-derived products. Due to the proximity in chemical shift of the pyrrolidine ring resonances and their extended reciprocal coupling, the ${ }^{1} \mathrm{H}$ NMR spectrum (Figure 2) shows numerous overlapped multiplets, as further shown in the 2D-NMR COSY spectra (see the Supporting Information). 2D-NMR NOESY experiments (see the Supporting Information) revealed intense NOE cross-couplings between naphthyl protons and the $\mathrm{H}^{\mathrm{b}, \mathrm{c}}$ protons and also the $\mathrm{H}^{\mathrm{a}}$ proton. Because of the facile pyramidal inversion that occurs at the nitrogen atom of the five-membered ring, these products are present as single racemic stereoisomers and no syn-anti combinations are possible. ${ }^{[27]}$

We also investigated the use of aromatic aldehydes in place of paraformaldehyde, together with sarcosine and VBP, to 


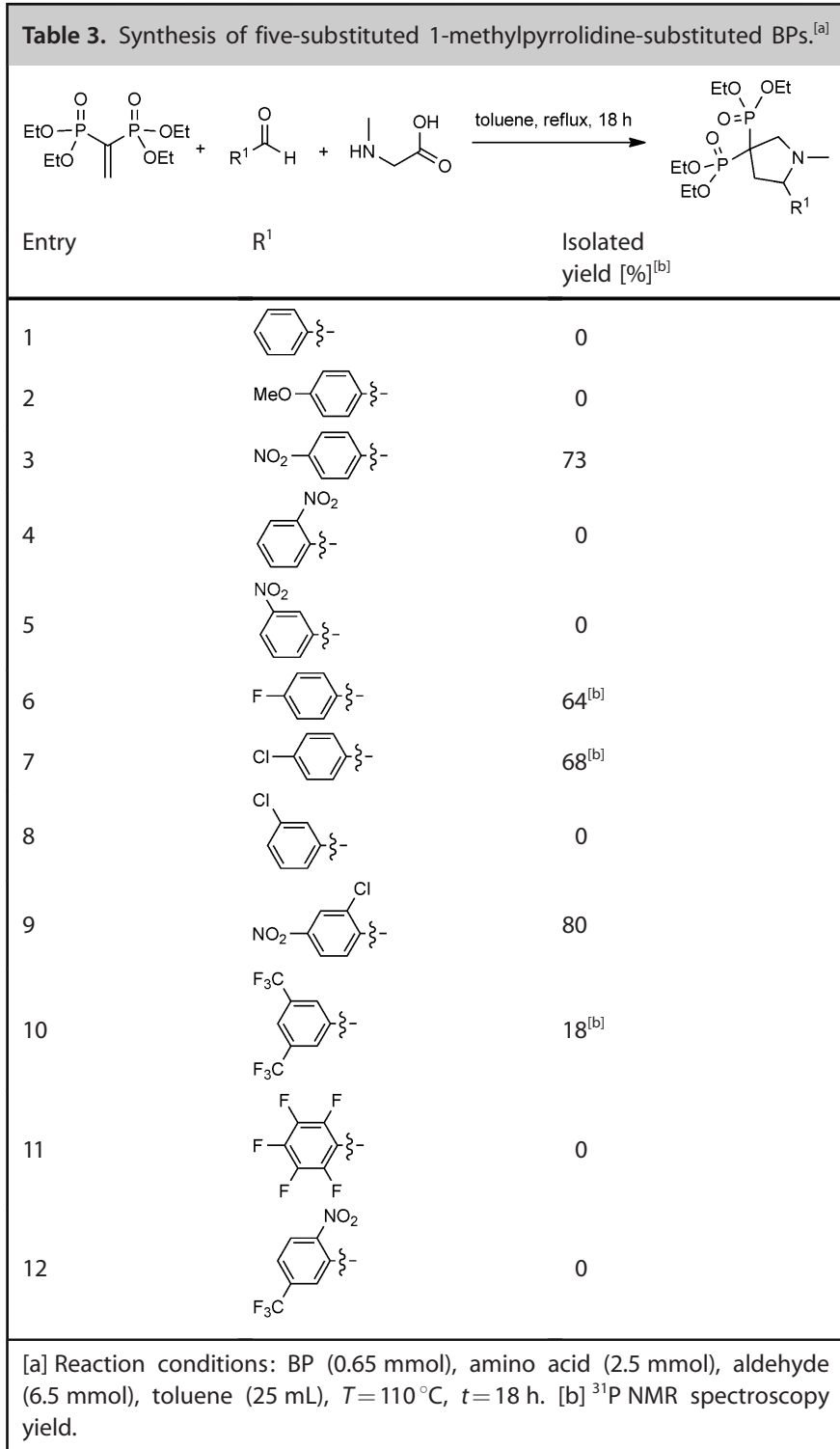

obtain two- or five-substituted 1-methylpyrrolidine BP derivatives (Table 3).

The reaction proved to be very sensitive to the electronic properties of the aromatic aldehydes. In fact, benzaldehydes and $p$-methoxybenzaldehyde did not show formation of the desired product (Table 3, entries 1 and 2), whereas electronpoor aldehydes, such as $p$-chlorobenzaldehyde, $p$-fluorobenzaldehyde, and $p$-nitrobenzaldehyde, provided the corresponding products in 68,64, and $73 \%$ yield, respectively (Table 3, entries 3,6 , and 7). Even the position of the substituent group on the ring affected the reaction and resulted in product formation only when the EWG was present in the para position (Table 3, entries 3, 6, 7, 9). EWG substituents in the meta or ortho positions led to moderate yields (Table 3, entry 10) or did not react at all (Table 3, entries 4, 5, 8). Other aldehydes, such as pentafluorobenzaldehyde (Table 3 , entry 11 ) or 2-nitro-5-trifluoromethylbenzaldehyde (Table 3, entry 12 ), even if electron poor, did not provide the corresponding cycloadducts with VBP.
An attempt to use a more elaborate carbonyl compound to implement in the pyrrolidine ring was carried out by replacing the aromatic aldehyde with isatin in the reaction with sarcosine and VBP. Isatin is a particularly attractive building block in the synthesis of both natural products and heterocyclic and noncyclic compounds in enantioselective reactions ${ }^{[28]}$ and in the preparation of biologically active spirooxindoles. ${ }^{[29]}$ The reaction between VBP, sarcosine, and isatin led to the formation of the corresponding tetraethyl-1'-methyl-2-oxospiro[indolyn$3,2^{\prime}$-pyrrolidin]-4',4'diyldiphosphonate in $51 \%$ yield in $18 \mathrm{~h}$ (Scheme 2).
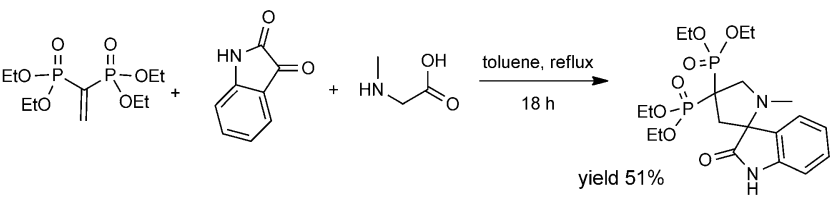

Scheme 2. Synthesis of tetraethyl-1'-methyl-2-oxospiro[indolyn-3,2'-pyrrolidin]-4', 4'-diyldiphosphonate.

Because the free bisphosphonic acid is the active form in this class of drug candidates, as an example, tetraethyl-1-methylpyrrolidine-3,3-diyldiphosphonic ester was subjected to deprotection of the ethyl ester moieties by treatment with bromo trimethylsilane followed by hydrolysis with wet methanol. The reaction proceeded nicely and provided the corresponding water-soluble 1-methylpyrrolidine-3,3-diyldiphosphonic acid in $>98 \%$ yield, which thus confirmed the stability of the pyrrolidine BP units under the deprotection conditions.

\section{Conclusion}

We have reported an extensive investigation of the synthesis of a new class of N-substituted heterocyclic BPs with pyrrolidines units, obtained by a 1,3-dipolar cycloaddition between azomethine ylides and BP precursors. The methodology led to the efficient preparation of a wide range of (1-methylpyrrolidine-3,3-diyl)bis(phosphonic acid) derivatives with different substituents in position 4.

As general trends, we observed that the synthesis is more productive with sarcosine than with other amino acids in which the $-\mathrm{NH}_{2}$ group has no substituents. Similarly, better results were obtained by starting from BP precursors with either low steric hindrance or electron-withdrawing substituents. The same steric and electronic trends were observed with respect to aldehydes or carbonyl compounds (isatin).

With the appropriate combination of aldehyde, prochiral BP, and amino acid, this process allows us to obtain, with a fair degree of flexibility, N-heterocyclic pyrrolidine bisphosphonates, similar to the highly active, commercial zoledronate, with the possibility to modulate the structure (albeit with some limitations), in particular in positions 1, 4, and 5 of the amino BP ring.

Finally, as we have proved with the simplest homologue in this series, the deprotection of the ethyl ester moieties with tri- 
methylsilyl bromide and subsequent hydrolysis occurs rather simply, which provides high yields of the free bisphosphonic acid in its biologically active form.

These molecules are currently under study to investigate their biological and toxicological properties.

\section{Experimental Section}

\section{Reagents and materials}

All reagents employed were commercial products (from Aldrich) and were used without purification. Vinylidenebisphosphonate tetraethyl ester (VBP) was prepared as reported in the literature. ${ }^{[13]}$ The prochiral vinylidenebisphosphonate tetraethyl esters were obtained by treating methylene bisphosphonate with aromatic aldehydes. ${ }^{[30]}$ All the synthetic work was carried out in air without the exclusion of atmospheric oxygen. Solvents were dried and purified according to standard methods. Flash chromatography was performed on 230-400 mesh silica and thin-layer chromatography was carried out on $20 \times 20 \mathrm{~cm}$ ALUGRAM $^{\circledR}$ Xtra SIL G/UV254 Macherey-Nagel.

${ }^{1} \mathrm{H},{ }^{31} \mathrm{P}\left\{{ }^{1} \mathrm{H}\right\}$, and ${ }^{13} \mathrm{C}\left\{{ }^{1} \mathrm{H}\right\}$ NMR spectra were recorded at $298 \mathrm{~K}$ by using a Bruker Avance 300 spectrometer operated at 300.15, 121.5, and $75 \mathrm{MHz}$, respectively, unless otherwise stated. $\delta$ values [ppm] are relative to $\mathrm{Si}\left(\mathrm{CH}_{3}\right)_{4}$ or $85 \% \mathrm{H}_{3} \mathrm{PO}_{4}$. GC-MS analyses were performed by using a GC Trace GC 2000 coupled with a quadrupole MS Thermo Finnigan Trace MS and using the Full Scan method.

\section{General procedure for the synthesis of pyrrolidine-substitut- ed BPs}

Bisphosphonate precursor $(0.65 \mathrm{mmol})$ and toluene $(25 \mathrm{~mL})$ were added to a round-bottomed flask equipped with a reflux condenser and a magnetic stir-bar, then amino acid ( $2.5 \mathrm{mmol}, 4$ equiv.) and aldehyde ( $6.5 \mathrm{mmol}, 10$ equiv) were added. The mixture was heated at reflux for $18 \mathrm{~h}$ with vigorous stirring. The solvent was removed under reduced pressure and the reaction product was isolated by using column chromatography (eluent: 1:1 acetone/ethyl acetate) and characterized by using ${ }^{1} \mathrm{H},{ }^{31} \mathrm{P}$, and ${ }^{13} \mathrm{C}$ NMR spectroscopy and GC-MS analysis.

\section{Acknowledgements}

We thank the Italian MIUR and Università Ca' Foscari for financial support.

Keywords: cycloaddition $\cdot$ phosphorous $\cdot$ pyrrolidine $\cdot$ ylides

[1] S. Zhang, G. Gangal, H. Uludağ, Chem. Soc. Rev. 2007, 36, 507-531.

[2] R. G. G. Russell, Bone 2011, 49, 2-19.

[3] F. H. Ebetino, A.-M. L. Hogan, S. Sun, M. K. Tsoumpra, X. Duan, J. T. Triffitt, A. A. Kwaasi, J. E. Dunford, B. L. Barnett, U. Oppermann, M. W. Lundy, A. Boyde, B. A. Kashemirov, C. E. McKenna, R. G. G. Russell, Bone 2011, 49, 20-33.

[4] M. J. Rogers, J. C. Crockett, F. P. Coxon, J. Mönkkönen, Bone 2011, 49, $34-41$.

[5] Y. Zhang, R. Cao, F. Yin, M. P. Hudock, R.-T. Guo, K. Krysiak, S. Mukherjee, Y.-G. Gao, H. Robinson, Y. Song, J. H. No, K. Bergan, A. Leon, L. Cass, A.
Goddard, T.-K. Chang, F.-Y. Lin, E. Van Beek, S. Papapoulos, A. H.-J. Wang, T. Kubo, M. Ochi, D. Mukkamala, E. Oldfield, J. Am. Chem. Soc. 2009, $131,5153-5162$

[6] A. J. Wiemer, J. S. Yu, L. W. Shull, R. J. Barney, B. M. Wasko, K. M. Lamb, R. J. Hohl, D. F. Wiemer, Bioorg. Med. Chem. 2008, 16, 3652-3660.

[7] A. G. Roth, D. Drescher, Y. Yang, S. Redmer, S. Uhlig, C. Arenz, Angew. Chem. Int. Ed. 2009, 48, 7560-7563; Angew. Chem. 2009, 121, $7697-$ 7700.

[8] Y. Zhang, R. Cao, F. Yin, F.-Y. Lin, H. Wang, K. Krysiak, J.-H. No, D. Mukkamala, K. Houlihan, J. Li, C. T. Morita, E. Oldfield, Angew. Chem. Int. Ed. 2010, 49, 1136-1138; Angew. Chem. 2010, 122, 1154-1156.

[9] J. H. No, F. de Macedo Dossin, Y. Zhang, Y.-L. Liu, W. Zhu, X. Feng, J. Anny Yoo, E. Lee, K. Wang, R. Hui, L. H. Freitas-Junior, E. Oldfield, Proc. Natl. Acad. Sci. USA 2012, 109, 4058-4063.

[10] R. L. McConnell, H. W. Coower, J. Am. Chem. Soc. 1956, 78, 4450-4452.

[11] G. R. Kieczykowski, R. B. Jobson, D. G. Mellilo, D. F. Reinhold, V. J. Grenda, I. Shinkai, J. Org. Chem. 1995, 60, 8310-8312.

[12] M. Egorov, S. Aoun, M. Padrines, F. Redini, D. Heymann, J. Lebreton, M. Mathé-Allainmat, Eur. J. Org. Chem. 2011, 7148-7154.

[13] R. A. Nugent, M. Murphy, S. T. Schlachter, C. J. Dunn, R. J. Smith, N. D. Staite, L. A. Galinet, S. K. Shields, D. G. Aspar, K. A. Richard, N. A. Rohloff, J. Med. Chem. 1993, 36, 134-139.

[14] D. Simoni, N. Gebia, F. P. Invidiata, M. Eleopra, P. Marchetti, R. Rondanin, R. Baruchello, S. Provera, C. Marchioro, M. Tolomeo, L. Marinelli, V. Limongelli, E. Noveliono, A. Kwaasi, J. Dunford, S. Buccheri, N. Cacamo, F. Dieli, J. Med. Chem. 2008, 51, 6800-6807.

[15] P. C. B. Page, M. J. McKenzie, J. A. GAlagher, J. Org. Chem. 2001, 66, 3704-3708.

[16] D. Granchi, A. Scarso, G. Bianchini, A. Chiminazzo, A. Minto, P. Sgarbossa, R. A. Michelin, G. Di Pompo, S. Avnet, G. Strukul, Eur. J. Med. Chem. 2013, 65, 448-455.

[17] A. Chiminazzo, L. Sperni, M. Damuzzo, G. Strukul, A. Scarso, ChemCatCh em 2014, 6, 2712-2718.

[18] G. Bianchini, A. Scarso, A. Chiminazzo, L. Sperni, G. Strukul, Green Chem. 2013, 15, 656-662.

[19] M. Ferrer-Casal, A. P. Barboza, S. H. Szajnman, J. B. Rodriguez, Synthesis 2013, 45, 2397-2404.

[20] R. Ruzziconi, G. Rici, A. Gioiello, H. Couthon-Gourvès, J.-P. Gourvès, J. Org. Chem. 2003, 68, 736-742.

[21] O. Bortolini, I. Mulani, A. De Nino, L. Maiuolo, M. Nardi, B. Russo, S. Avnet, Tetrahedron 2011, 67, 5635-5641.

[22] a) B. J. Foust, C. Allen, S. A. Holstein, D. F. Wiemer, Bioorg. Med. Chem. 2016, 24, 3734-3741; b) X. Zhou, J. E. Reilly, K. A. Loerch, R. J. Hohl, D. F. Wiemer, Beilstein J. Org. Chem. 2014, 10, 1645-1650.

[23] R.-Y. Zhu, C.-S. Wang, F. Jiang, F. Shi, S.-J. Tu, Tetrahedron: Asymmetry 2014, 25, 617-624.

[24] O. Tsuge, S. Kanemasa, Adv. Heterocycl. Chem. 1989, 45, 231 -349.

[25] The reaction of proline with aromatic aldehydes and tetraethyl vinylidenebisphosphonate (VBP), catalyzed by cerium(IV) oxide, to form 3-substituted-hexahydro-1H-pyrrolizine-1,1-diyl-bis(phosphonate) products was reported, see: G. Li, M. Wu, D. Kong, R. Liu, X. Zhou, F. Liu, New J. Chem. 2014, 38, 3350-3353.

[26] J. Adrio, J. C. Carretero, Chem. Commun. 2014, 50, 12434-12446.

[27] A. R. Katritzky, C. A. Ramsden, J. A. Joule, V. V. Zhdankin, "Handbook of Heterocyclic Chemistry", Elsevier, Oxford, 2010.

[28] M. Flores, J. Pena, P. García-García, N. M. Garrido, D. Diez, Curr. Org. Chem. 2013, 17, 1957-1985.

[29] M. M. M. Santos, Tetrahedron 2014, 70, 9735 - 9757.

[30] C. Yuan, J. Yao, S. Li, Phosphorus Sulfur Silicon Relat. Elem. 1990, 53, 21

Manuscript received: December 16, 2016

Accepted Article published: January 11, 2017

Final Article published: February 9, 2017 